Supplement of

\title{
Human amplified changes in precipitation-runoff patterns in large river basins of the Midwestern United States
}

Sara A. Kelly et al.

Correspondence to: Sara A. Kelly (sara.kelly@aggiemail.usu.edu)
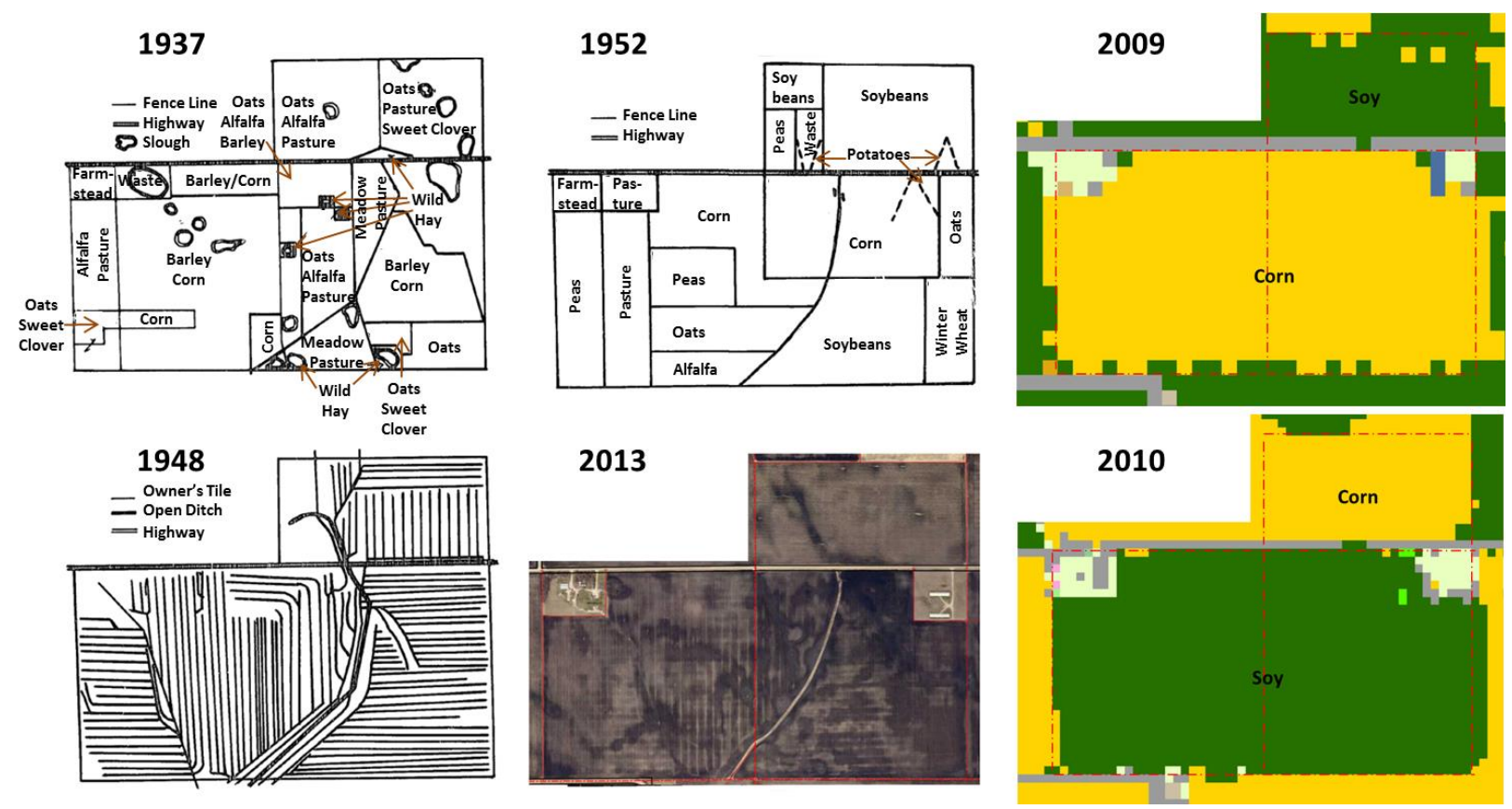

Figure S1: Field land use and tile arrangement before (1937) and after (1952) tile installation (1948) near Mapleton, MN (adapted from Burns, 1954); aerial photograph flown in spring 2013 shows the modern tile pattern remains relatively unchanged with a corn-soybean crop rotation (2009-2010), from the Cropland Data Layer. 


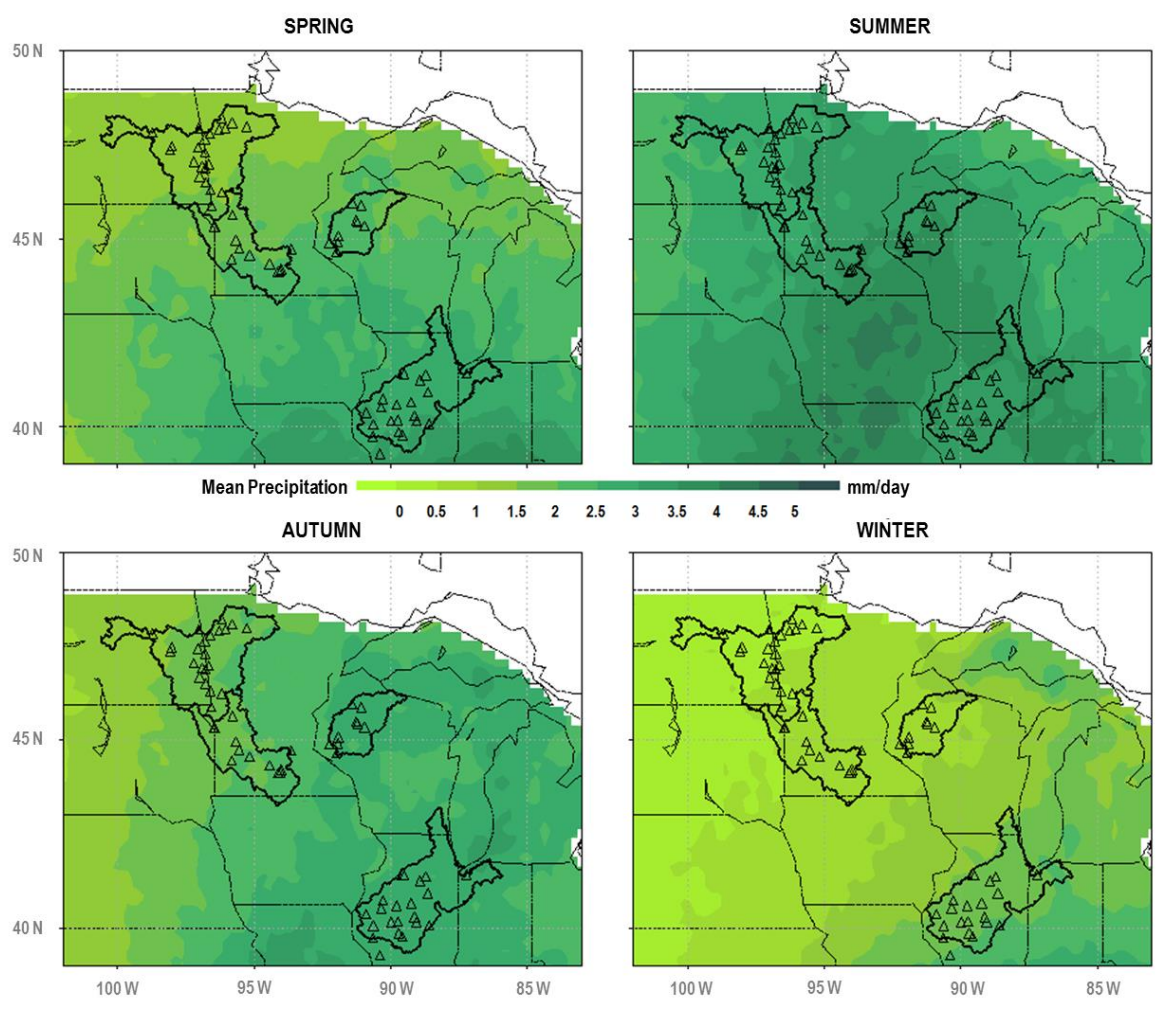

Figure S2. Seasonally averaged long term daily precipitation means (1981-2010) across the Upper Midwest: spring (MAM), summer (JJA), autumn (SON), and winter (DJF); USGS gage locations (Table 1) indicated by open triangles.

Comparison of monthly precipitation total reported as an average depth (cm) from PRISM (used in this study) and Livneh et al. (2013) (L13) for each watershed (Figure S3). If PRISM and L13 precipitation depths were equivalent in every month, then all points would plot on the 1:1 line. On average (1935-2011) the difference between the two monthly precipitation datasets is $1 \%$ for each study watershed.

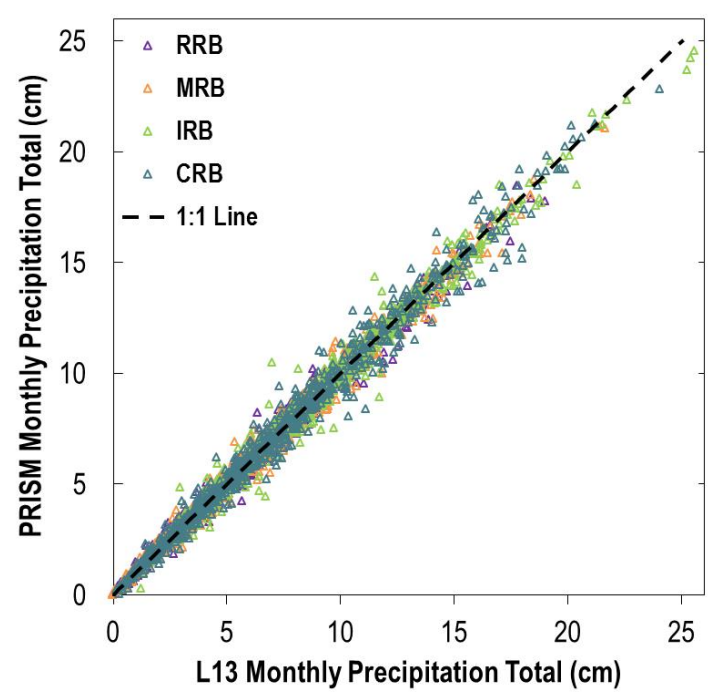

Figure S3. Spatially averaged, total monthly $(\mathrm{cm})$ precipitation $(1935-2011)$ for each watershed from PRISM and Livneh et al. 2013 plotted with 1:1 line. 
Figure S4 shows a comparison of monthly (March-November during 2001-2011) $\mathrm{ET}_{\mathrm{a}}$ estimates produced by Livneh et al. (2013) (L13) with $\mathrm{ET}_{\mathrm{p}}$ estimates (available from: http://agwx.soils.wisc.edu/uwex_agwx/sun_water/et_wimn) produced following the methods of Diak et al. (1998) (D98) for a location in the MRB (44 N, $94 \mathrm{~W}$ ) and the CRB $(45.2 \mathrm{~N}, 91.6 \mathrm{~W})$. On average, the estimates of $\mathrm{ET}_{\mathrm{a}}$ are $19 \%$ (raw) and $26 \%\left(17 \%\right.$ reduction in JJA ET ${ }_{\mathrm{a}}$ ) lower than estimates of $\mathrm{ET}_{\mathrm{p}}$ in the MRB, and 16\% (raw) and 24\% (17\% reduction in JJA ET $\mathrm{E}_{\mathrm{a}}$ ) lower than estimates of $\mathrm{ET}_{\mathrm{p}}$ in the CRB.

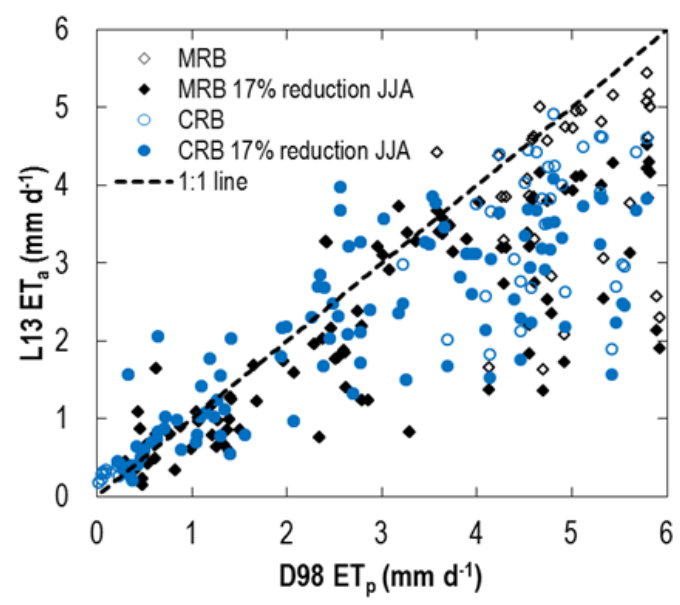

Figure S4. Monthly (March-November) average daily $\left(\mathrm{mm} \mathrm{d}^{-1}\right)$ estimates of $\mathrm{ET}_{\mathrm{p}}$ following methods of Diak et al., 1998 versus estimates of $\mathrm{ET}_{\mathrm{a}}$ from Livneh et al., 2013 during 2001-2011.

Figure S5 shows average monthly ETa from Livneh et al. (2013) compared against four AmeriFlux sites near the study watersheds (Table 2) as well as data from Bryan et al. (2015). In general, the L13 data show an earlier peak in ETa for the cropland sites in Rosemount, MN and Bondville, IL, and overestimate average annual ETa by 17\% (raw) and 7\% (17\% reduction in JJA) for Bondville and 14\% (raw) and 5\% (17\% reduction in JJA) for Rosemount. The L13 data overestimate ETa at Willow Creek, WI (broadleaf deciduous forest) by as much as $31 \%$ (raw) and $19 \%$ (17\% reduction in JJA) annually, and underestimate ETa at Brookings, SD (grassland) by 29\% (raw) and 34\% (17\% reduction in JJA) annually.
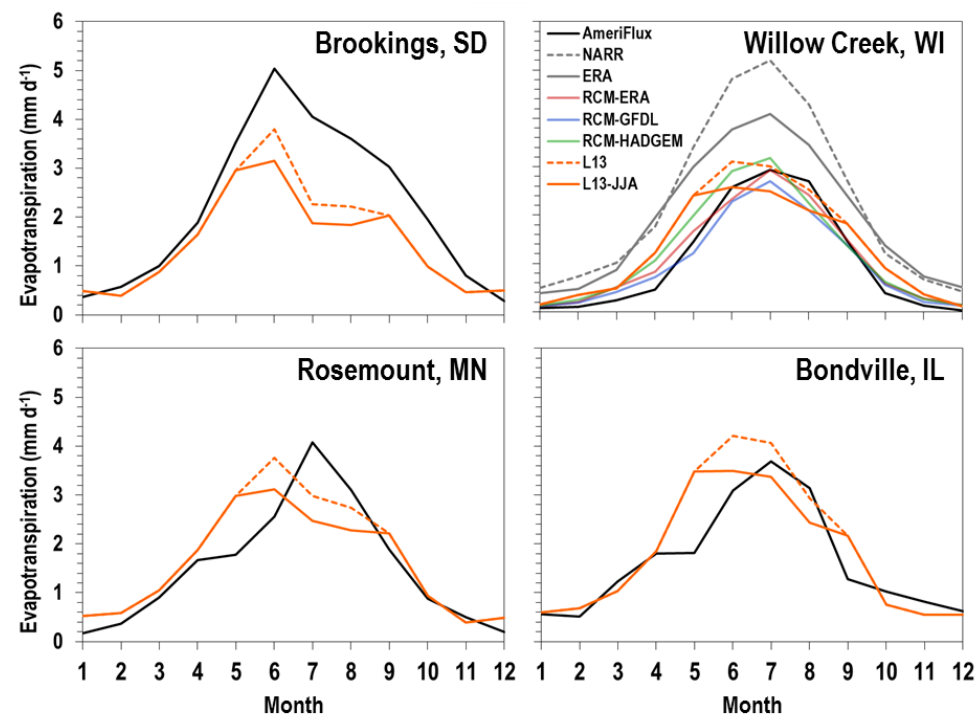

Figure S5. Average monthly evapotranspiration rate $\left(\mathrm{mm} \mathrm{d}^{-1}\right)$ at four AmeriFlux sites (see Table 2) compared to modeled evapotranspiration rates used in this study (L13 \& L13-JJA) and Bryan et al. 2015. 


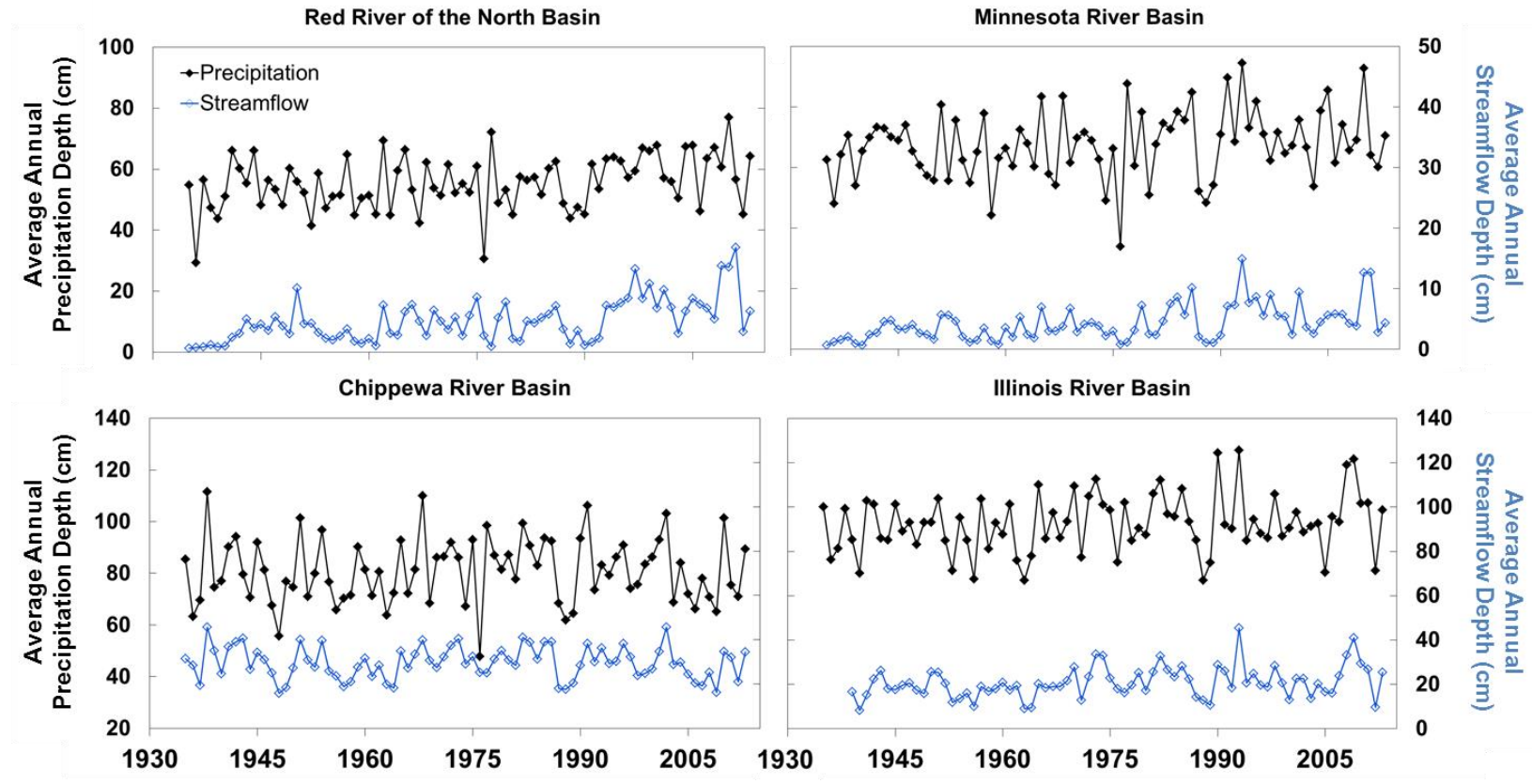

Figure S6. Annual, spatially averaged watershed precipitation and streamflow depths (cm) for each study basin.

Table S1. Resulting p-values of 572 statistical tests (t-test and KS-test) comparing pre-period and post-period flow and precipitation based on the 1974/1975, piecewise linear regression (PwLR), and land cover transition (LCT) breakpoints for each basin (Table 3). P-values are highlighted based on their significance: bolded values are p-values with $95 \%$ confidence level or greater, grey values are p-values with less than a $95 \%$ confidence level, and black values are p-values where significance depends on the breakpoint. Italicized grey values reported for the CRB are not reliable because the post-period includes fewer than 10 years of data.

\begin{tabular}{|c|c|c|c|c|c|c|c|c|c|c|c|c|c|}
\hline & \multicolumn{3}{|c|}{ Flow: t-test } & \multicolumn{3}{|c|}{ Flow: KS-test } & \multicolumn{3}{|c|}{ Precipitation: t-test } & \multicolumn{3}{|c|}{ Precipitation: KS-test } \\
\hline & & $74 / 75$ & PwLR & LCT & $74 / 75$ & PwLR & LCT & $74 / 75$ & PwLR & LCT & $74 / 75$ & PwLR & LCT \\
\hline \multirow{13}{*}{ CRB } & January & 0.341 & 0.893 & 0.846 & 0.653 & 0.958 & 0.902 & 0.278 & 0.097 & 0.214 & 0.223 & 0.050 & 0.082 \\
\hline & February & 0.372 & 0.680 & 0.851 & 0.449 & 0.878 & 0.953 & 0.337 & 0.039 & 0.309 & 0.446 & 0.071 & 0.367 \\
\hline & March & 0.566 & 0.871 & 0.525 & 0.219 & 0.749 & 0.205 & 0.188 & 0.369 & 0.574 & 0.234 & 0.348 & 0.700 \\
\hline & April & 0.468 & 0.267 & 0.719 & 0.506 & 0.152 & 0.416 & 0.192 & 0.277 & 0.258 & 0.169 & 0.308 & 0.575 \\
\hline & May & 0.826 & 0.485 & 0.264 & 0.482 & 0.311 & 0.622 & 0.933 & 0.374 & 0.187 & 0.906 & 0.697 & 0.445 \\
\hline & June & 0.900 & 0.552 & 0.211 & 0.908 & 0.628 & 0.142 & 0.833 & 0.434 & 0.117 & 0.945 & 0.587 & 0.246 \\
\hline & July & 0.706 & 0.775 & 0.308 & 0.584 & 0.893 & 0.606 & 0.463 & 0.609 & 0.358 & 0.567 & 0.794 & 0.360 \\
\hline & August & 0.174 & 0.508 & 0.364 & 0.354 & 0.450 & 0.200 & 0.496 & 0.945 & 0.769 & 0.760 & 1.000 & 0.856 \\
\hline & September & 0.517 & 0.990 & 0.723 & 0.357 & 0.958 & 0.654 & 0.286 & 0.912 & 0.752 & 0.657 & 0.925 & 0.654 \\
\hline & October & 0.103 & 0.778 & 0.593 & 0.110 & 0.887 & 0.817 & 0.022 & 0.026 & 0.304 & 0.097 & 0.073 & 0.423 \\
\hline & November & 0.240 & 0.894 & 0.713 & 0.337 & 0.887 & 0.902 & 0.510 & 0.905 & 0.806 & 0.375 & 0.944 & 0.874 \\
\hline & December & 0.263 & 0.973 & 0.806 & 0.387 & 0.971 & 0.931 & 0.380 & 0.135 & 0.062 & 0.337 & 0.175 & 0.045 \\
\hline & Annual & 0.499 & 0.793 & 0.340 & 0.721 & 0.918 & 0.291 & 0.243 & 0.571 & 0.295 & 0.246 & 0.764 & 0.614 \\
\hline
\end{tabular}




\begin{tabular}{|c|c|c|c|c|c|c|c|c|c|c|c|c|c|}
\hline & & & & & \multirow[b]{2}{*}{0.136} & & \multirow[b]{2}{*}{0.543} & & \multirow[b]{2}{*}{0.454} & & \\
\hline \multirow{13}{*}{ IRB } & January & 0.123 & 0.030 & 0.092 & & 0.079 & 0.265 & & 0.250 & 0.529 & & 0.425 & 0.211 \\
\hline & February & 0.355 & 0.082 & 0.184 & 0.353 & 0.043 & 0.216 & 0.224 & 0.108 & 0.764 & 0.433 & 0.359 & 0.899 \\
\hline & March & 0.035 & 0.062 & 0.045 & 0.104 & 0.114 & 0.099 & 0.649 & 0.777 & 0.619 & 0.619 & 0.836 & 0.828 \\
\hline & April & 0.174 & 0.438 & 0.158 & 0.335 & 0.479 & 0.353 & 0.780 & 0.832 & 0.588 & 0.883 & 0.947 & 0.638 \\
\hline & May & 0.182 & 0.344 & 0.155 & 0.126 & 0.398 & 0.161 & 0.212 & 0.113 & 0.326 & 0.063 & 0.063 & 0.138 \\
\hline & June & 0.105 & 0.077 & 0.280 & 0.082 & 0.071 & 0.117 & 0.798 & 0.742 & 0.845 & 0.811 & 0.643 & 0.954 \\
\hline & July & 0.451 & 0.411 & 0.525 & 0.518 & 0.436 & 0.614 & 0.453 & 0.585 & 0.214 & 0.585 & 0.519 & 0.443 \\
\hline & August & 0.090 & 0.249 & 0.212 & 0.181 & 0.508 & 0.259 & 0.054 & 0.408 & 0.257 & 0.037 & 0.475 & 0.108 \\
\hline & September & 0.004 & 0.062 & 0.009 & 0.003 & 0.111 & 0.041 & 0.511 & 0.465 & 0.118 & 0.685 & 0.728 & 0.113 \\
\hline & October & 0.074 & 0.147 & 0.065 & 0.082 & 0.139 & 0.142 & 0.142 & 0.072 & 0.363 & 0.113 & 0.143 & 0.378 \\
\hline & November & 0.034 & 0.007 & 0.041 & 0.075 & 0.008 & 0.074 & 0.023 & 0.004 & 0.109 & 0.053 & 0.013 & 0.045 \\
\hline & December & 0.021 & 0.011 & 0.010 & 0.040 & 0.019 & 0.022 & 0.122 & 0.081 & 0.039 & 0.136 & 0.203 & 0.050 \\
\hline & Annual & 0.011 & 0.019 & 0.017 & 0.012 & 0.018 & 0.020 & 0.086 & 0.075 & 0.085 & 0.117 & 0.141 & 0.183 \\
\hline \multirow{13}{*}{ MRB } & January & 0.000 & 0.000 & 0.000 & 0.001 & 0.000 & 0.000 & 0.096 & 0.520 & 0.182 & 0.369 & 0.672 & 0.338 \\
\hline & February & 0.000 & 0.000 & 0.000 & 0.002 & 0.000 & 0.000 & 0.722 & 0.842 & 0.659 & 0.540 & 0.938 & 0.604 \\
\hline & March & 0.007 & 0.005 & 0.002 & 0.089 & 0.055 & 0.074 & 0.017 & 0.394 & 0.060 & 0.107 & 0.515 & 0.148 \\
\hline & April & 0.041 & 0.039 & 0.016 & 0.011 & 0.034 & 0.003 & 0.159 & 0.164 & 0.239 & 0.489 & 0.344 & 0.684 \\
\hline & May & 0.000 & 0.001 & 0.000 & 0.001 & 0.001 & 0.001 & 0.716 & 0.366 & 0.469 & 0.807 & 0.657 & 0.636 \\
\hline & June & 0.000 & 0.000 & 0.000 & 0.000 & 0.000 & 0.000 & 0.500 & 0.957 & 0.418 & 0.807 & 0.879 & 0.677 \\
\hline & July & 0.002 & 0.007 & 0.000 & 0.021 & 0.020 & 0.003 & 0.300 & 0.107 & 0.151 & 0.351 & 0.230 & 0.223 \\
\hline & August & 0.008 & 0.012 & 0.000 & 0.017 & 0.040 & 0.001 & 0.239 & 0.641 & 0.133 & 0.097 & 0.806 & 0.064 \\
\hline & September & 0.017 & 0.062 & 0.001 & 0.106 & 0.225 & 0.015 & 0.224 & 0.077 & 0.242 & 0.334 & 0.112 & 0.261 \\
\hline & October & 0.002 & 0.001 & 0.000 & 0.012 & 0.007 & 0.002 & 0.082 & 0.015 & 0.029 & 0.115 & 0.088 & 0.116 \\
\hline & November & 0.001 & 0.000 & 0.000 & 0.006 & 0.000 & 0.001 & 0.262 & 0.418 & 0.380 & 0.260 & 0.519 & 0.445 \\
\hline & December & 0.000 & 0.000 & 0.000 & 0.002 & 0.000 & 0.000 & 0.435 & 0.138 & 0.385 & 0.608 & 0.457 & 0.413 \\
\hline & Annual & 0.000 & 0.000 & 0.000 & 0.001 & 0.001 & 0.000 & 0.033 & 0.072 & 0.011 & 0.098 & 0.199 & 0.051 \\
\hline \multirow{13}{*}{ RRB } & January & 0.000 & 0.000 & 0.005 & 0.005 & 0.000 & 0.013 & 0.117 & 0.169 & 0.412 & 0.112 & 0.105 & 0.368 \\
\hline & February & 0.000 & 0.000 & 0.016 & 0.003 & 0.000 & 0.034 & 0.155 & 0.321 & 0.050 & 0.246 & 0.326 & 0.183 \\
\hline & March & 0.006 & 0.012 & 0.171 & 0.011 & 0.005 & 0.217 & 0.050 & 0.108 & 0.021 & 0.062 & 0.247 & 0.054 \\
\hline & April & 0.069 & 0.079 & 0.036 & 0.105 & 0.102 & 0.115 & 0.981 & 0.902 & 0.619 & 0.974 & 0.823 & 0.574 \\
\hline & May & 0.016 & 0.003 & 0.004 & 0.059 & 0.013 & 0.014 & 0.321 & 0.039 & 0.046 & 0.312 & 0.129 & 0.186 \\
\hline & June & 0.015 & 0.000 & 0.001 & 0.038 & 0.002 & 0.001 & 0.170 & 0.138 & 0.351 & 0.105 & 0.032 & 0.569 \\
\hline & July & 0.000 & 0.000 & 0.001 & 0.001 & 0.000 & 0.005 & 0.288 & 0.251 & 0.886 & 0.244 & 0.418 & 0.943 \\
\hline & August & 0.000 & 0.000 & 0.013 & 0.000 & 0.000 & 0.043 & 0.687 & 0.551 & 0.681 & 0.598 & 0.650 & 0.786 \\
\hline & September & 0.002 & 0.000 & 0.024 & 0.002 & 0.000 & 0.084 & 0.094 & 0.036 & 0.047 & 0.009 & 0.013 & 0.081 \\
\hline & October & 0.003 & 0.000 & 0.010 & 0.003 & 0.002 & 0.053 & 0.010 & 0.015 & 0.002 & 0.011 & 0.003 & 0.004 \\
\hline & November & 0.000 & 0.000 & 0.001 & 0.000 & 0.000 & 0.003 & 0.409 & 0.560 & 0.918 & 0.270 & 0.341 & 0.943 \\
\hline & December & 0.000 & 0.000 & 0.000 & 0.001 & 0.000 & 0.013 & 0.487 & 0.058 & 0.000 & 0.639 & 0.071 & 0.002 \\
\hline & Annual & 0.000 & 0.000 & 0.000 & 0.000 & 0.000 & 0.005 & 0.019 & 0.004 & 0.009 & 0.010 & 0.008 & $\mathbf{0 . 0 2 7}$ \\
\hline
\end{tabular}

